\title{
Accurate Calculation of Fringe Fields in the LHC Main Dipoles
}

\author{
S. Kurz \\ ITE, University of Stuttgart, Germany \\ S. Russenschuck, N. Siegel \\ CERN, Geneva, Switzerland
}

\begin{abstract}
The ROXIE program developed at CERN for the design and optimization of the guperconducting LHC magnets hes been recently extended in a collaboration with the University of Stuttgart, Germany, with a fleld computation method based on the coupling between the boundary element (BFM) and the flnite element (FEM) technique. This avoids the meshing of the coils and the air regions, and avoids the artificial far field boundary conditions. The method is therefore specially suited for the accurate calculation of fields in the superconducting magnets in whlch the field is dominated by the coil. We will present the fringe field calculations in both 2d and ad geometries to evaluate the effect of connections and the cryostat on the fheld quality and the flux density to which auxiliary bus-bars are exposed.
\end{abstract}

\section{INTRODUCTION}

'The design and optimization of the LHC magnets is governed by the requirement of an extremely uniform field which is mainly defined by the layout of the superconductIng coils. Even very small geometrical effects such as the keystoning of the cable, the insulation, grading of the current density in the cable due to different cable compaction and coil deformations due to collaring, cool down and electromagnetic forces have to be considered for the field calculation. For the field optimization of the LHC magnets [1], the usefulness of commercial software has shown to be limited in particular in the three dimensional case. Therefore the ROXIE [2] program package was doveloped at CERN for the design and optimization of the LHC superconductIng magnets. Furthermore, the application of the BEMFEM coupling method [3] yiolds the reduced field due to the magnetization of the iron yoke anly. This avoids the meshing of the coils and the air regions, and avoids the artificial far field boundary conditions. The method is therefore specially suited for the calculation of fringe fields in the superconducting magnets, both in 2 and 3 dimensions.

\section{ThE BEM-FEM COUPLING ME'THOD}

The total magnetic induction $\vec{B}$ in a certain point $\vec{\xi}$ in the aperture of the magnet can be decomposed into a contribution $\vec{B}_{S}$ due to the superconducting coil and a contribution $\vec{B}_{\mathbb{R}}$ duo to the magnetic yoke. If the fields are expressed in terms of the magnetic vector potential $\vec{B}=\operatorname{curl} \vec{A}$, then the decomposition into source and reduced contributions gives

$$
\vec{A}=\overrightarrow{A_{S}}+\vec{A}_{\mathrm{R}} \text {. }
$$

This approach has the following intrinsic advantages:

Manuscript received September 26, 1999
- The coil field can be taken into account in terms of its source vector potential $\vec{A}_{\mathrm{S}}$, which can be obtained easily from the filamentary currents $I_{S}$ by means of Biot-Savart type integrals without meshing of the coil.

- The BEM-FEM coupling method allows a direct computation of the reduced vector potential $\vec{A}_{\mathrm{R}}$ instead of the total vector potential $\vec{A}$. Consequently, numerical errors do not influence the dominating contribution $\vec{A}_{\mathrm{S}}$ due to the superconducting coil.

- Since the field in the aporture is calculated through the integration over all the BEM elements, local field errors in the iron yoke cancel out and the calculated multipole content is sufficiently accurate even for a very sparso mesh. - The surrounding air region need not be meshed at all. This simplifies the preprocessing and avoids artificial boundary conditions at some "far" distance. Since the air region is not meshed, only the iron region meshing is affected by geometrical modifications. This strongly supports the feature based, parametric geomotry modelling which is required for mathematical optimization.

The elementary model problem for a single aperture model dipole (featuring both Dirichlet and Neumann bounds on the iron yoles) is shown in Fig. 1. When the

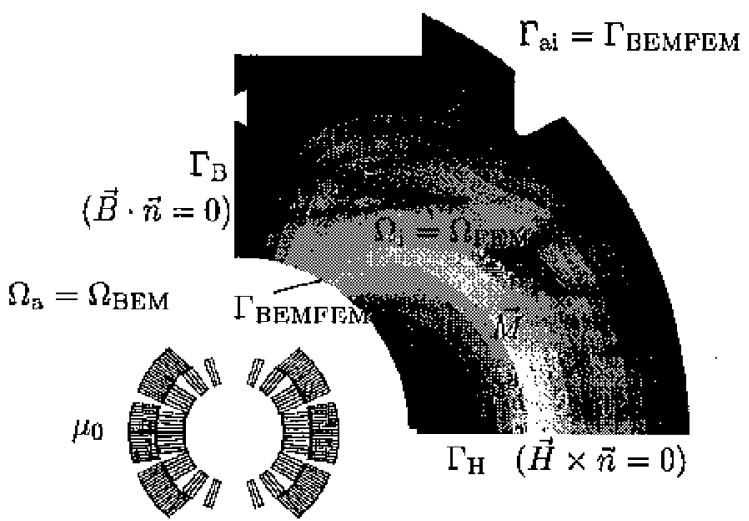

Fig. 1. Elementary model problem for the numerical fiold calculation of a superconducting (single aperture) modlol magnet. In the iron domain the total vector potential is displayed. The nonconductive air region $\Omega_{\mathrm{B}}$ containg a certain number of conductor sources $\vec{J}$ which do not intersect the iron region $\Omega_{\text {f }}$. The finiteelement method inside the magnetic body $\Omega_{\mathrm{i}}=\Omega_{F E M}$ is coupled with the boundary-element mothod in the domain outside the magnetic material $\Omega_{\mathrm{a}}=\Omega_{\mathrm{BEM}}$, by means of the normal derivetive of the vector-potential on the interface $\Gamma_{\mathrm{a}}=\Gamma_{\mathrm{BEMFEM}}$ between iron and air. 
BEM-FEM coupling method is applied, only the magnetic sub-domain $\Omega_{\mathrm{i}}$ which coincides with the magnetic yoke has to be meshed by finite elements. Iron saturation effects can then be treated within the FEM domain. The nonmagnetic sub-domain $\Omega_{\mathrm{a}}$ which represents the surrounding air region and the excitation coil is treated by the boundary element method. Only the common boundary $\Gamma_{\mathrm{ai}}$ needs to be discretized by boundary elements. If the domain $\Omega_{\mathrm{i}}$ is discretized into finite elements ( $C^{b}$-contimous, isoparar metric 20-noded hexahedron elements are used), and the Galerkin method is applied to the weak formulation, then a non linear system of equations is obtained

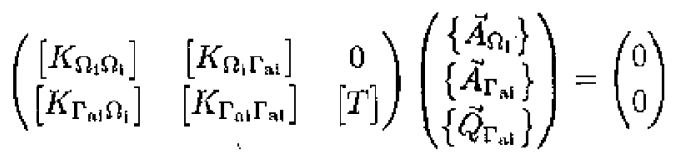

with all nodal values of $\vec{A}_{\Omega_{1}}, \vec{A}_{\Gamma_{n 1}}$ and $\vec{Q}_{\Gamma_{\mathrm{an}}}$ grouped in arrays. As we will see later, $\vec{Q}_{\Gamma_{\text {al }}}$ is the normal derivative of $\vec{A}$ on $\Gamma_{\text {ai }}$

$$
\vec{Q}_{\Gamma_{\mathrm{a}}}=-\frac{\partial A_{\Gamma_{\mathrm{n}}}^{\mathrm{BEM}}}{\partial n_{\mathrm{a}}}
$$

which we assume as given. The subscripts $\Gamma_{\text {ai }}$ and $\Omega_{\mathrm{i}}$ refer to nodes on the boundary and in the interior of the domain, respectively. The domain and boundary integrals in the weak formulation yield the stiffness matrices $[K]$ and the boundary matrix $[T]$. The stiffress matrices depend on the local permeability distribution in tho nonlinear material. All the matrices in (2) are sparse. For the meshing of the boundary $\Gamma_{\mathrm{aj}}$ with boundary elements $\Gamma_{\mathrm{a} i, \mathrm{j}}, C^{\mathrm{C}}$-continuous, isoparametric 8-noded quadrilateral boundary elements are used. The discrete analogue of the Fredholm integral equation can be obtained by atccessively putting the evaluation point $\overrightarrow{r_{0}}$ at the location of each nodal point $\overrightarrow{r_{j}}$. This procedure is called point-wise collocation and yields a linear system of equations,

$$
[G]\left\{\vec{Q}_{\Gamma_{\mathrm{a}}}\right\}+[H]\left\{\vec{A}_{\Gamma_{\mathrm{Bl}}}\right\}=\left\{\vec{A}_{\mathrm{a}}\right\} .
$$

In $(4),\left\{\overrightarrow{A_{s}}\right\}$ contains the values of the source vector potential at the nodal points $\vec{r}_{j}, j=1,2, \ldots$ The matrices $[G]$ and $[H]$ are unsymmotric and fully populated. An overall numerical description of the field problem can be obtained by complementing the FEM description (2) by the BEM doscription (4) resulting in

$$
\left(\begin{array}{ccc}
{\left[K_{\Omega_{1} \Omega_{1}}\right]} & {\left[K_{\Omega_{1} \Gamma_{\mathrm{al}}}\right]} & 0 \\
{\left[K_{\Gamma_{\mathrm{a} 1} \Omega_{\mathrm{i}}}\right]} & {\left[K_{\Gamma_{\mathrm{a} 1} \Gamma_{\mathrm{a} i \mathrm{i}}}\right]} & {[T]} \\
0 & {[H]} & {[G]}
\end{array}\right)\left(\begin{array}{c}
\left\{\vec{A}_{\Omega_{1}}\right\} \\
\left\{\vec{A}_{\Gamma_{\mathrm{al}}}\right\} \\
\left\{\vec{Q}_{\mathrm{F}_{\mathrm{gi}}}\right\}
\end{array}\right)=\left(\begin{array}{c}
0 \\
0 \\
\left\{\vec{A}_{\mathrm{s}}\right\}
\end{array}\right)
$$

Equation (4) gives exactly the missing relationship between the Dirichlet data $\left\{\vec{A}_{\Gamma_{+1}}\right\}$ and the Neumann data $\left\{\vec{Q}_{\Gamma_{\mathrm{i} 1}}\right\}$ on the boundary $\Gamma_{\text {ai }}$. It can be shown [4] that this procedure yields the correct physical interface conditions, the continuity of $\vec{n} \cdot \vec{B}$ and $\vec{n} \times \vec{H}$ across $\Gamma_{\text {ai }}$.

A detailed description of the method and the application to superconducting magnets can be found in [4], [5],

\section{IIL. Field Quality in Accelerator Magnets}

The magnetic field errors in the aperture of the magnets are expressed as the coefficients of the Fourier-sories oxpansion of the radial field component at a given reference radius (in the 2-dimensional case). In the 3 -dimensional case, the trensverse field components are given at a longitudinal position $z_{0}$ or integrated over the entire length of the magnet. For a given radial component of the magnetic flux density $B_{r}$ at a reference radius $r=r_{0}$ inside the aperture of a magnet the Fourier-series expansion of the field reads

$$
\begin{array}{r}
B_{r}\left(r_{0}, \varphi\right)=\sum_{n=1}^{\infty}\left(B_{n}\left(r_{0}\right) \sin n \varphi+A_{n}\left(r_{0}\right) \cos n \varphi\right) \\
B_{r}\left(r_{0}, \varphi\right)=B_{N}\left(r_{0}\right) \sum_{n=1}^{\infty}\left(b_{n}\left(r_{0}\right) \sin n \varphi+a_{n}\left(r_{0}\right) \cos n \varphi\right)
\end{array}
$$

with the main field component $B_{N}(N=1$ dipole, $N=$ 2 quadrupole, etc.). The $B_{n}$ are called the normal and the $A_{n}$ the shew components of the field given in Tesla, $b_{n}$ the normal relative, and $a_{n}$ the skew relative field components. They are dimensionless and are usually given in units of $10^{-4}$ at a $17 \mathrm{~mm}$ reference radius. In practice the $B_{r}$ components are calculated in discrete points $\varphi_{k}=\frac{k \pi}{P}-\pi, k=0,1,2, \ldots, 2 P_{-1}$ in the interval $[-\pi, \pi)$ and a discrete Fourier transform is carried out:

$$
\begin{aligned}
& A_{n}\left(r_{0}\right) \approx \frac{1}{P} \sum_{k=0}^{2 P-1} B_{r}\left(r_{0}, \varphi_{k}\right) \cos n \varphi_{k} \\
& B_{n}\left(r_{0}\right) \approx \frac{1}{P} \sum_{k=0}^{2 P-1} B_{r}\left(r_{0}, \varphi_{k}\right) \sin n \varphi_{k} .
\end{aligned}
$$

The interpolation-error depends on the number of evaluation points and the amount of higher order multipole orrors in the field. For the calculation of multipoles up to the order $n=13,79$ evaluation points ( $P=40$ ) are sufficient.

\section{.IV. REsults}

\section{A. Field Quality in Collored Coils}

Space limitations in the existing LEP tunnel and economical considerations have dictated a so-called two-inone magnet design with two sets of coils and beam channels within a common mechanical structure, iron yoko, and cryostat. As a ifst example of the application of the BEMFEM method we calculate the magnetic field in a configuration as shown in Fig. 2. Using double aperture stainlesssteel collars with a relative permeability of $\mu_{\mathrm{r}}=1.0025$ creates asymmetries in the magnetic field in the case of warm measurements of the collared coil assembly where only one aperture is powered. The relative feld errors at $17 \mathrm{~mm}$ reference radius in the aperture are given in Table 1. As was mentioned earlier tho BEM-FEM method does not require the meshing of the coil (which can therefore be modelled with the required accuracy) and does not require a "far field" boundary condition which would influence considerably the results of this unbounded field problem. 


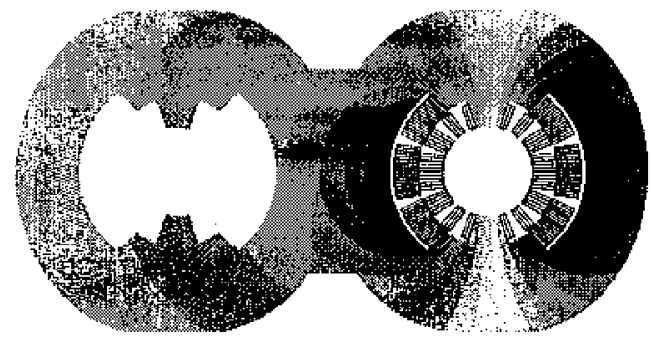

Fig. 2. Geometric model of one dipole coil poweted for warrn measurement in the combined collar structure assumirg a constent relative permeability of $\mathrm{AH}_{\mathrm{r}}=1: 0025$. The figure displeys the magnetic fiux density in the collars.

\section{TABLE I}

ADDITIONAL FIELO FRRORS IN COLL COLLAR. ABSEMBLY WITH RUGHT-HAND-SIDE APERTURE POWERED FOR WARM MEASUREMENTS. (UNTTS OF $10^{-4}$ AT 17 MM.) AND NOMINAL VALUES FOR A BARE COIL WITHOUT COLLAR.

\begin{tabular}{cccc|cccc}
\hline \multicolumn{4}{c}{ Nominal } \\
\hline$b_{2}$ & 0.0000 & $b_{6}$ & 0.0000 & $b_{2}$ & -0.0737 & $b_{6}$ & 0.0000 \\
$b_{3}$ & 3.9150 & $b_{7}$ & 0.7456 & $b_{3}$ & -1.6326 & $b_{7}$ & -0.0918 \\
$b_{4}$ & 0.0000 & $b_{8}$ & 0.0000 & $b_{4}$ & -0.0018 & $b_{8}$ & 0.0000 \\
$b_{5}$ & -1.0385 & $b_{9}$ & 0.1224 & $b_{5}$ & 0.4306 & $b_{9}$ & 0.0081 \\
\hline
\end{tabular}

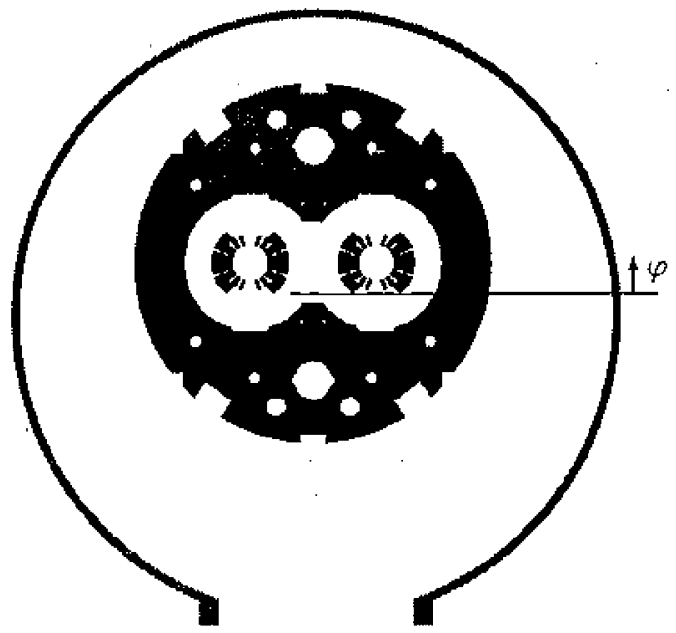

Fig. 3. Geometric model of the two-dimensional cross-section of the magnet showing iron yoke and cryostat vacium vesse].

\section{B. Infuence of the Cryostat Eccentricity}

The cold mass of the two-in-one magnet is placed in the vacuum vessel of the cryostat (made of magnetic steel) with an off-centering of $80 \mathrm{~mm}$ in the vertical plane, see Fig. 3 .
Fig. 4. Fringe field outgide the vacuum vessel as a function of the angular position $\varphi$.

TABLE II

At the position of the support posts of the cold mass a circular opening is made in the cryostat vacuum vessel, closed by a non-magnetic plate. As a first approach, this opening has been modelled as a two-dimensional problem. The fringe field is higher at the opening but stays below $0.5 \cdot 10^{-3}$ Tesla, see Fig. 4. Again, the advantage of the BEM-FEM method lays in the fact that the surrounding air regions do not have to be considered and that the disconnected iron parts can be meshed independently with the required refinement. The eccentricity of the cryostat can therefore easily be modelled. Tablo 2 gives the nominal, and the additional field errors due to the cryostat at nominal fiold of $8.36 \mathrm{~T}$ in units of $10^{-4}$ at $17 \mathrm{~mm}$.

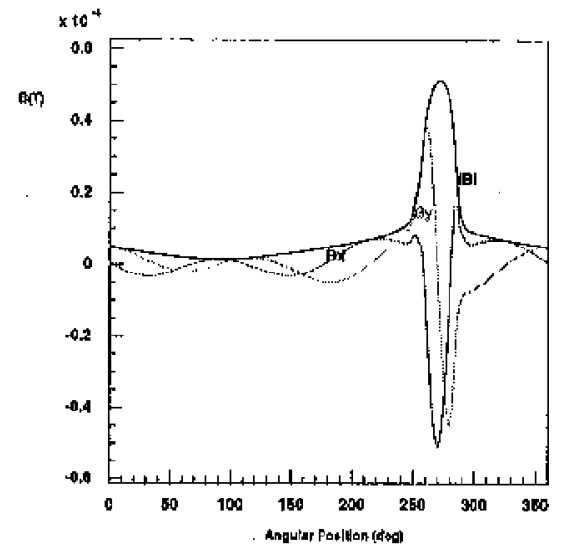

ADDITIONAL FIELD ERTORS DUE TO ASYMMETRY OF THE VAOUUM VESSEL AT NOMINAL FIELD IN UNITS OF $10^{-4}$ AT 17 MM AND NOMINAL ERRORS FOR A COLD-MASS WTTHOUT VACUUM VESSEL.

\begin{tabular}{cccc|cccc}
\hline & \multicolumn{3}{c}{ Nominal } & \multicolumn{4}{c}{ Additional } \\
\hline & & $a_{1}$ & 0.000 & & & $a_{1}$ & 0.422 \\
$b_{2}$ & 0.646 & $a_{2}$ & 0.000 & $b_{2}$ & 0.099 & $a_{2}$ & \\
$b_{3}$ & 5.775 & $a_{3}$ & 0.000 & $b_{3}$ & 0.015 & $a_{3}$ & 0.016 \\
$b_{4}$ & 0.217 & $a_{4}$ & 0.000 & $b_{4}$ & 0.005 & $a_{4}$ & -0.002 \\
$b_{5}$ & -0.914 & $a_{5}$ & 0.000 & $b_{5}$ & 0.000 & $a_{5}$ & 0.000 \\
\hline
\end{tabular}

\section{Fringe Field in the Coil-end Region}

In order to reduce the peak field in the coil-end and thus increase the quench margin in the region with a weaker mechanical structure, tho magnetic iron yoke ends approximately $80 \mathrm{~mm}$ from the onset of the coil-end at the nonconnection side and about $300 \mathrm{~mm}$ from the onset of the coil-end at the connection end. This asymmetry results from the aim of a further reduction of the field in the ramp and splice region. The BEM-FEM coupling method is used importance for not having to mesh the coil becomes obvious from Fig. 5. The ends can be modelled with the required for the calculation of the end-fields. In particular in 3D, the 
accuracy as the source field can be calculated directly by means of Blot-Savart's law. The two-in-one magnet end configuration with the common yoke used for the computation is shown in Fig. 6. Fig. 7 shows the field components

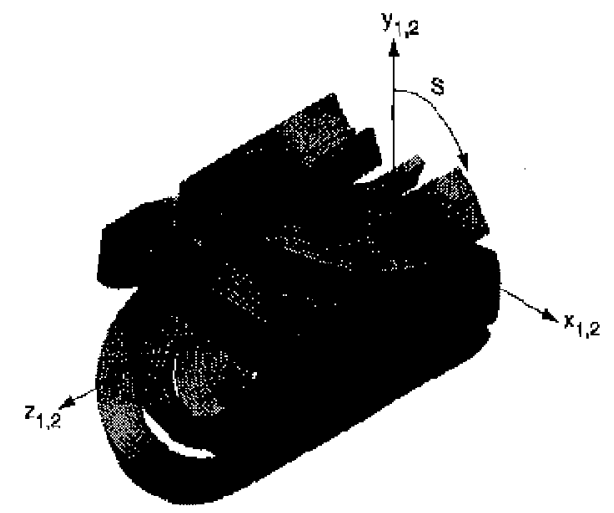

Fig. 5. Geometric model of the dipole coil-end with loca! coordinate system.

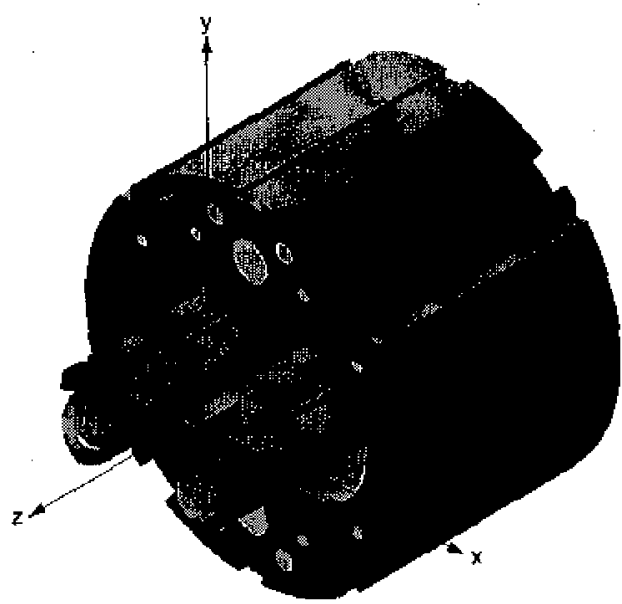

Fig. 6, Full 3D modgl of coils and two-jn-one iron yoke. The location of the bus-bar is at $x=285 \mathrm{~mm}$ and $y=-67 \mathrm{~mm}$.

along a line in the end-region of the twin-aperture dipole prototype magnet (MBP2) from $z=-500 \mathrm{~mm}$ (inside the magnet yoke) to $z=400 \mathrm{~mm}$ outside the yoke. The onset of the coil-ends are at $z=0$. On the non-connection side the iron yoke ends at $z=-80 \mathrm{~mm}$, on the connection side the iron yoke ends at $z=-300 \mathrm{~mm}$. This calculation is important for the evaluation of the forces acting on the 600 $A$ and $6 \mathrm{kA}$ auxiliary bus bars.

\section{CONCLUSION}

The BEM-FEM method is specially well suited for the calculation of fringe fields in superconducting magnets, as the coils and the air regions do not have to be represented in the finite-element mesh, discretization errors only influence the calculation of the yoke magnetizetion and there is not an artificial "far field" boundary condition. The method has been illustrated by three examples, the results of which
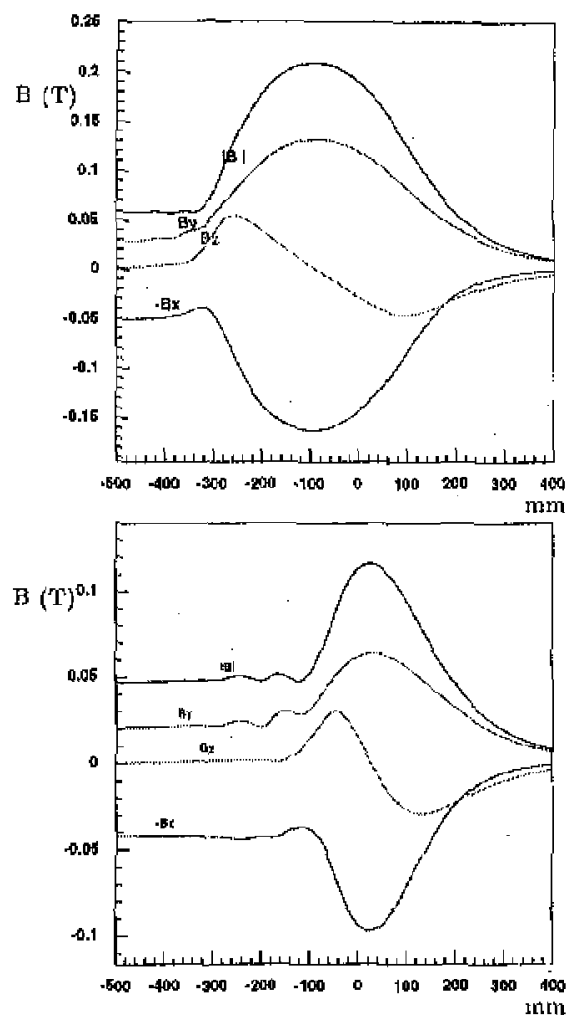

Fis. 7. Fringo field outside the dipole cold mass near the coil-end region, Top: connection end, Bottom: return end.

can be summarized as follows: 1) For warm measurements, an additional field error due to the stainless-sted collar of about -1.6 units in the relative $b_{3}$ component has to be considered. 2) The fringe field outside the cryostat stays below $5 \cdot 10^{-4} \mathrm{~T}$ and 3 ) the fleld seen by the $600 \mathrm{~A}$ and $6 \mathrm{kA}$ auxiliary bus bars stays below $0.21 \mathrm{~T}$ in the coil-end region.

\section{REFERENCES}

[1] The LAIC study group. LHC, The Large Hadron Collider, Conceptual Design. CERN/AC/95-05 (LHC), 1995.

[2] Editor: Stephan Russenschuck. ROXIE: Routine for the Optimization of magnet X-sections, Inverse field calculation and coil End design. Yellow Report, OERN 99-01([SBN=92-9083-140-5), 1999.

(3) J. Fetzer, S. Abele, and G. Lehner. Die Koppluns der Randelementmethode und der Methode der finiten Elemente zur Lösung drejdimensionaler elektromagnetischer Feldproblerne atf unendlichem Grundgebiet. Archiv für Elehtrotechnik, 76(5):361368,1993

(4) S. Kurz, J. Fetzer, and W. Rucker. Coupled BEM-FEM methode for 3D field calculations with iron saturation. Proceedings of the First International ROXIE users meeting and workhop, 1999.

(5) S. Kurz and S. Ruasenschuck. The application of the BEM-FEM coupling method for the accurate calculation of fields in superconducting magnets. Accepted for publication in Electrical Engineering. 